\title{
NEUROEVOLUTIONARY APPROACH TO CONTROL OF COMPLEX MULTICOORDINATE INTERRELATED PLANTS
}

\author{
Yuriy Kondratenko 1), Oleksiy Kozlov 1), Oleksandr Gerasin ${ }^{2)}$ \\ 1) Petro Mohyla Black Sea National University, 10 68th Desantnykiv st., Mykolaiv, 54003, Ukraine \\ ykondrat2002@yahoo.com,yuriy.kondratenko@chmnu.edu.ua,kozlov_ov@ukr.net \\ 2) Admiral Makarov National University of Shipbuilding, 9 Heroes of Ukraine ave., Mykolaiv, 54025, Ukraine, \\ oleksandr.gerasin@nuos.edu.ua
}

Paper history:

Received 18 June 2019

Received in revised form 25 August 2019

Accepted 02 December 2019

Available online 31 December 2019

\section{Keywords:}

multicoordinate interrelated plant;

automatic control system;

neural controller;

neural network;

neuroevolution;

genetic algorithm;

caterpillar mobile robot.

\begin{abstract}
This paper presents the developed by the authors step-by-step neuroevolution based approach to designing control systems for complex multicoordinate interrelated plants (MIP). The proposed approach allows us to build the structure of the automatic control system (ACS) for the MIP on the basis of the single complex neural controller (NC) with multiple inputs and outputs as well as to implement the effective training of its multilayer neural network (NN) by means of the evolutionary based algorithm, taking into account the mutual influence of all variables of the MIP in an optimal way. In order to study and validate the efficiency of the presented approach the design of the ACS for the spatial motion of caterpillar mobile robot (MR) able to move on inclined and vertical ferromagnetic surfaces is carried out in this work. The developed ACS based on the NC with optimal structure allows us to achieve high quality indicators of spatial motion control, taking into account the mutual influence of control channels of the MR's speed and angle that confirms the high efficiency of the proposed approach.
\end{abstract}

Copyright $(\mathbb{C}$ Research Institute for Intelligent Computer Systems, 2019. All rights reserved.

\section{INTRODUCTION}

Nowadays, there is a high level of automation of all involved technological processes and technical nodes at any stage of the material (substance) processing in the various sectors of industry, energetics and transportation $[1,2]$. The use of highly effective automatic control systems of the technical plants is caused by the need of increasing their productivity, economic and operational indicators as well as the level of quality of the technological processes in general $[3,4]$.

One of the most important challenges of automation of different technological processes and technical plants is the implementation of proper control of their main variables in order to stabilize the optimal operating modes under conditions of various types of disturbing influences [5]. Usually, the majority of technical plants and units, found today in the modern industry and transport areas, are complex non-stationary and/or nonlinear control objects, the operating conditions of which may change in rather wide limits [6-8]. Practice shows that automatic control of such plants based on conventional control principles in many cases does not allow achieving enough high quality indicators of control [9]. Also, the complexity of the given above problem additionally increases due to the fact that a sufficiently large number of industrial and transport plants are multicoordinate interrelated plants $[10,11]$. Internal combustion engines and hydraulic drives, chemical reactors, industrial and mobile robots, unmanned underwater and aerial vehicles, machines of metallurgical industry as well as other dynamic plants in which two or more coordinates are simultaneously controlled are the examples of such plants $[2,7,12-14]$. Thus, to solve this problem and improve the quality of indicators and total effectiveness of MIPs control systems it is expedient to apply approaches and means of advanced control theory based on artificial intelligence and soft computing, in particular, neural 
networks, fuzzy systems and bioinspired algorithms [15-17].

The issues of development and application of intelligent neural, fuzzy and neuro-fuzzy control systems of various complex plants are widely covered in scientific literature [18-21]. Analysis of the current research in this area shows, that neural controllers are most often used in automation systems of complex nonlinear multidimensional plants operating under conditions of uncertain disturbances, and for which there is no sufficient manual control experience gained by their operators [22-24]. This is confirmed by many examples of their successful application presented in a number of works, in particular, in control systems of different types of industrial and mobile robots [25, 26], DC and synchronous motors [27, 28], power and heating plants [29-31], ships [32] and others [33, 34].

The neural controllers' performance in the control systems of the complex plants is directly dependent on the effectiveness of the training methods used $[15,17]$. Contemporary studies show that evolutionary bioinspired approaches and algorithms have proven themselves to be quite promising at training, synthesis and optimization of neural networks and other intelligent systems [17, $35,36]$. An approach to neural networks training by means of various evolutionary methods, in particular, genetic algorithms (GA) is called neuroevolution [37-39] and has the following advantages over conventional methods of training: (a) does not require training samples in the form of input-output pairs; (b) allows finding the global optimum for solving a problem, not getting stuck in local minima; (c) does not impose any restrictions on the fitness functions [40-42].

A number of studies are currently underway to develop automation systems for different types of industrial plants based on a neuroevolutionary approach, the results of which are presented in publications [43-46]. In particular, the development of neural network controller for a fuel cell turbine hybrid energy system via multi-objective neuroevolution and the Pareto concavity elimination transformation is presented in [43]. The example of the efficient usage of neuroevolutionary algorithms for training the optimal impedance controller for robotic manipulator is shown in [44]. In turn, paper [45] presents the neuroevolutionary approach to solve the problem of stochastic inventory control in multi-echelon systems. Also, the deep neuroevolutionary control approach is proposed and applied to the autonomous vehicle driving control system in the paper [46].

As regards designing functional structures, control devices, mathematical models and synthesis methods of control systems for complex multicoordinate interrelated plants on the basis of neuroevolutionary techniques, this problem remains unresolved and is the subject of research by scientists around the world.

Therefore, the aim of this work is development and research of the neuroevolution based approach to design of automatic control systems for complex multicoordinate interrelated plants.

\section{CONTROL OF MULTICOORDINATE INTERRELATED PLANTS}

At designing of automatic control systems, it is often necessary to solve the problem of controlling of complex multi-input multi-output (MIMO) plants, characterized by many controlled quantities at the output [47]. In this case, a multi-channel control system should be created. The complexity of solving this problem largely depends on the features of a MIMO plant [10, 47].

At studying a MIMO plant, two cases are possible: (a) the output variables of the plant are not interrelated and a change in one value does not affect the others; (b) output variables are interrelated and a change in one of them leads to a change in the others [11].

In the first case, each control variable of the plant depends on only one control action and does not depend (or weakly depends) on the other control actions. For such systems, secondary connections can be neglected and the ACS is considered as one consisting of separate subsystems. Own regulator of a certain type is used to control each output variable of the plant. In this case, the control circuit of each output quantity is completely autonomous and can be considered as an independent ACS in the study. The control system is a multichannel ACS with independent control channels in this case.

The plant control variables substantially depend on different control actions in the second case. Now, the relations between the controlled quantities cannot be neglected, and the plant is considered to be multicoordinate and interrelated.

The functional structure of the ACS of the multicoordinate interrelated plant is presented in Fig. 1, where the following notations are accepted: $Y_{\mathrm{S} i}, i=\{1,2, \ldots, m\}$ are the set values of the controlled variables of the multicoordinate interrelated plant; $Y_{\mathrm{R} i}, \quad i=\{1,2, \ldots, m\}$ are the real values of the controlled variables of the MIP; $\varepsilon_{i}, i=\{1,2, \ldots, m\}$ are the control errors of the MIP coordinates; $\mathrm{C}_{i}$, $i=\{1,2, \ldots, m\}$ are separate controllers of the MIP coordinates; $u_{\mathrm{C} i}, i=\{1,2, \ldots, m\}$ are the output signals 
of the MIP controllers; $m$ is a total amount of the MIP controlled variables; $\mathbf{F}_{\mathrm{D}}$ is a vector of disturbances acting on the plant. Sensors and other peripheral control aids are not shown in this Figure.

The analysis of multicoordinate interrelated systems is rather difficult because of the complexity of the expressions, since the plant, in this case, is described by many transfer functions that take into account all the connections between $m$ controlled quantities, control actions $u_{\mathrm{C} i}, \quad i=\{1,2, \ldots, m\}$ and disturbances $\mathbf{F}_{\mathrm{D}}$ [46].

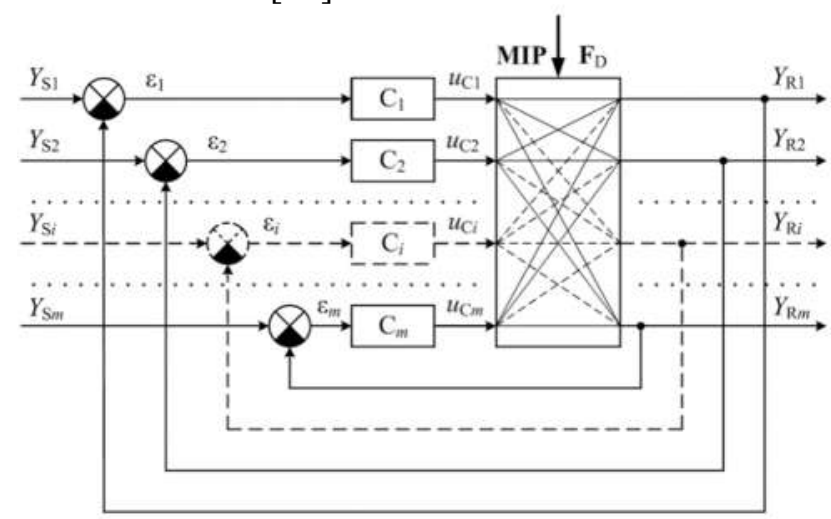

Figure 1 - Functional structure of the ACS of the multicoordinate interrelated plant

Multicoordinate interrelated systems tend to give the property of autonomy in practice [10]. The autonomous control is such a control, in which a change in any $i$-th regulated value does not change the other. Systems can be autonomous with respect to input or disturbing actions. The autonomy of the ACS can be achieved by implementation of specialized corrective feedbacks [47]. In this case, the ACS controllers $\mathrm{C}_{i}, \quad i=\{1,2, \ldots, m\}$ should be developed with additional connections between control channels to compensate their mutual influence. It is necessary to take into account the nature of the mutual influence of the controlled variables at designing the given controllers, and this is possible only if there is a deterministic description of this influence [11].

Mathematical models of the mutual influence of their controlled variables are not available or too complicated for a sufficiently large number of technical plants. Herewith, the controllers of their ACSs are developed separately for each variable (Fig. 1) on the basis of traditional methods, and the mutual influence of the controlled coordinates is considered to be disturbances and this does not allow obtaining sufficiently high quality indicators [47].

Thus, to increase the quality indicators and efficiency of the MIPs control systems it is advisable to use the proposed by the authors approach to ACSs development based on neuroevolution.

\section{NEUROEVOLUTION BASED APPROACH TO DESIGN OF CONTROL SYSTEMS FOR COMPLEX MIP}

The approach proposed by the authors is based on building the control system for the MIP on the basis of the single complex neural controller with multiple inputs and outputs. The main idea is that the complex multilayer neural network of the controller when properly trained takes into account the mutual influence of all variables of the MIP in an optimal way and allows implementing interconnected control with high quality indicators.

Thus, neuroevolution based approach to design of control systems for complex MIPs, proposed by the authors, consists of the following steps.

Step 1. Building of the ACS general structure on the basis of the single complex neural controller. At this stage, the structure of the control system is built, which is shown in Fig. 2. The given ACS has the complex NC, the number of outputs of which is equal to the number of controlled variables $m$. Wherein, the number of NC inputs $l$ is chosen so that $l \geq m$, since the controller inputs can be not only error signals $\varepsilon_{i}, \quad i=\{1,2, \ldots, m\}$, but also their derivatives of various orders and integrals $\left(\dot{\varepsilon}_{i}, \ddot{\varepsilon}_{i}, \ldots, \int \varepsilon_{i} d t\right)$. In turn, the complex $\mathrm{NC}$ in addition to the multilayer neural network includes units of preliminary calculation of derivatives of various orders and integrals from the input signals.

Also, parts of the given ACS structure are $m$ reference models (RM) of the control processes of MIP variables and comparing devices, which calculate the discrepancy $E_{i}$ between the RMs output signals $Y_{\mathrm{RM} i}$ and the real outputs of the plant $Y_{\mathrm{R} i}$, $i=\{1,2, \ldots, m\}$. In turn, the discrepancy $E_{i}$, $i=\{1,2, \ldots, m\}$ is used for the calculation of the total goal function value $J_{\Sigma}$ of the control system by means of the goal function calculation unit (GFCU).

Training of the complex $\mathrm{NC}$ of the control system in order to find the optimal value of the vector $\mathbf{X}_{\mathrm{NC}}$ of structural features and parameters of the multilayer neural network is implemented by the evolutionary based training mechanism (EBTM) using the current value of the total goal function $J_{\Sigma}$. In turn, EBTM applies sequential growth of layers and neurons to determine the optimal structure of the $\mathrm{NC}$ as well as genetic algorithm to find its optimal parameters (connections weights).

Step 2. Selection of structures and parameters of the reference models of the control processes of MIP variables. The structures and parameters of the reference models are selected at this stage depending on the desired transients of the MIP variables. For a sufficiently large number of controlled plants, the 
desired transient should be aperiodic in nature with a minimum duration $[2,4]$. Thus, the reference models for the MIP variables can be defined by the transfer functions $W_{\mathrm{RM} i}(\mathrm{~s})$ of the following form

$$
W_{\mathrm{RM} i}(\mathrm{~s})=\frac{Y_{\mathrm{RM} i}(\mathrm{~s})}{Y_{\mathrm{S} i}(\mathrm{~s})}=\frac{1}{\left(T_{\mathrm{RM} i} \mathrm{~s}+1\right)^{v_{i}}}, i=\{1, \ldots, m\},
$$

where $T_{\mathrm{RM} i}$ and $v_{i}$ are the time constant and order of the transfer function of the $i$-th RM.

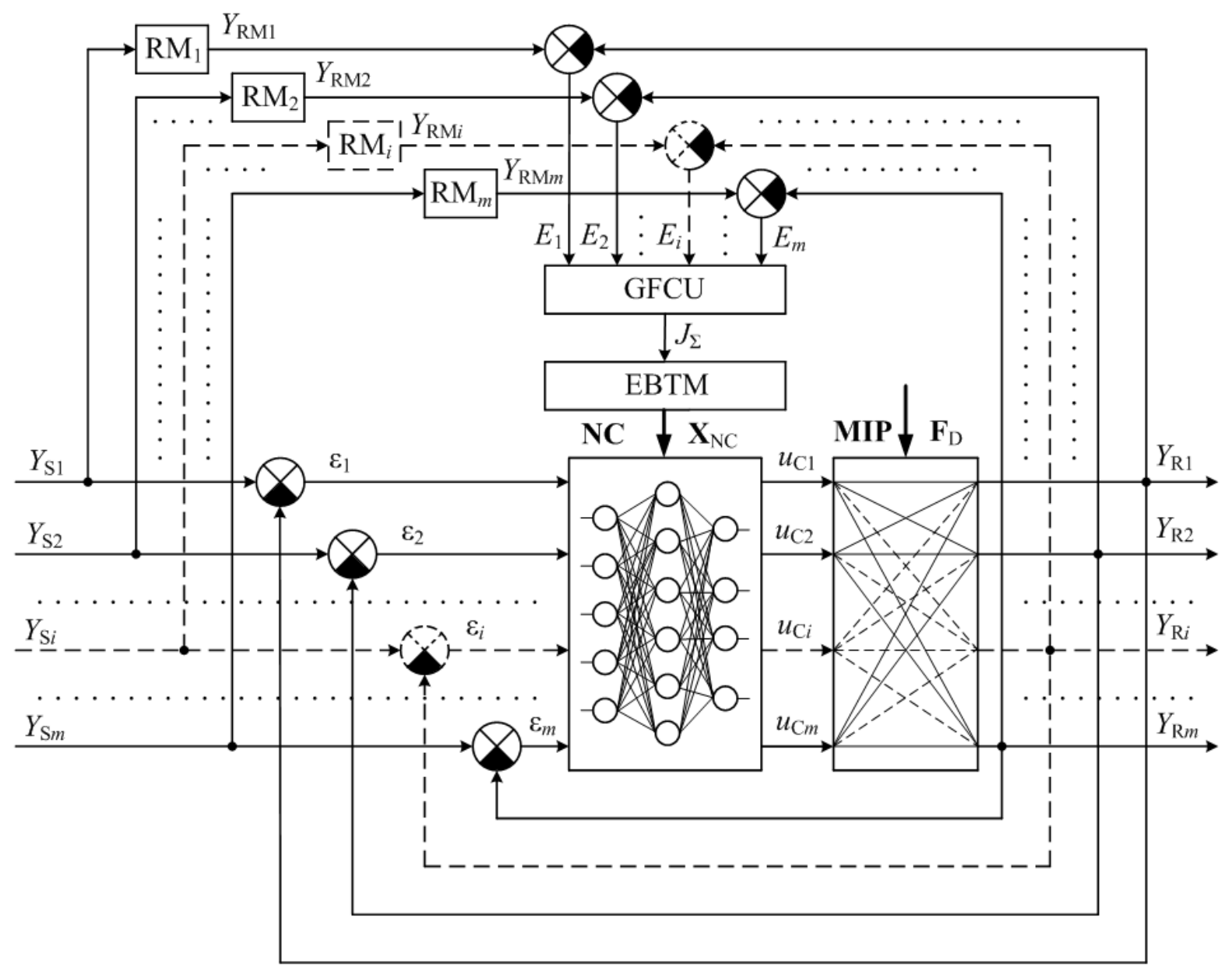

Figure 2 - General structure of the ACS of the MIP on the basis of the single complex neural controller

Step 3. Selection of structure, parameters and optimal value of the total goal function of the MIP control system. The structure, parameters and the optimal value of the total goal function $J_{\Sigma}$ of the MIP control system are selected at this stage. Wherein, the problem of synthesis of the structure and parameters of the neural network controller is a problem of multi-criteria optimization, in which the optimum of a set of goal functions is searched [17, 35]. In turn, the total goal function can be calculated differently in various problems of multi-criteria optimization. In this case, it is advisable to calculate the total goal function $J_{\Sigma}$ based on the method of aggregation functions [17] in the following way

$$
J_{\Sigma}=\sum_{i=1}^{m} k_{i} J_{i}, i=\{1, \ldots, m\}
$$

where $J_{i}$ is the goal function of the $i$-th controlled variable of the MIP; $k_{i}$ is the coefficient, that normalizes the value of the $i$-th goal function $J_{i}$ and determines the importance of control quality of the $i$ th controlled variable. For example, the mean integral quadratic error can be selected as the goal function of the $i$-th controlled variable, then the total goal function of the ACS has the form

$$
J_{\Sigma}=\sum_{i=1}^{m} \frac{k_{i}}{t_{\max }} \int_{0}^{t_{\max }} E_{i} d t, i=\{1, \ldots, m\}
$$

where $t_{\max }$ is the total time of the MIP transient.

In turn, the optimal value of the total goal function $J_{\text {Eopt }}$ is selected considering features of particular plant.

Step 4. Selection of initial structure and constraints of the multilayer neural network of the MIP control system. The initial structure and constraints of the multilayer neural network of the NC are selected at this stage. Since, the chromosomes size in the given neuroevolutionary 
approach directly depends on the number of connections weights and, accordingly, on the number of network layers and neurons in each layer, it is advisable to choose the most simple initial architecture of the neural network to reduce the computational costs during the GA search at Step 5 and then, if necessary, gradually increase the number of neurons and layers at Step 7 and Step 8 . Therefore, initially, three layers in the network should be set: input, one hidden layer and output. The number of neurons in the input and output layers are set equal to the number of inputs and outputs of the NC in accordance with its structure and the structure of the MIP control system. In turn, the number of neurons of the hidden layer can be set equal to the number of $\mathrm{NC}$ outputs. Also, the maximum possible number of hidden layers and neurons in a one hidden layer as well as interneuronal connections and type of activation functions are selected at this step. In turn, the constraints on the maximum possible number of hidden layers and neurons in a one hidden layer are selected in such a way that the synthesis process of the synaptic weights using GA with the corresponding maximum chromosomes size has acceptable computational and time costs.

Step 5. Synthesis of synaptic weights of the neural network for the selected architecture by means of $G A$. The search for optimal parameters of $\mathrm{NC}$ weights is carried out using genetic algorithm according to the following steps.

Step 5.1. Encoding of the NC network weights into the chromosome. The chromosome consists of genes, each of which is a NC network weight. Binary or real numbers can be used to represent genes [35]. For example, the synapse weights between the first input neuron and the first hidden layer neuron is encoded as $w_{11}$, between the second input and the third hidden neuron $-w_{23}$. In this case, all the synaptic weights between the input and the hidden layers become a vector $\mathbf{W}_{1}$, between the hidden and the output layers - vector $\mathbf{W}_{2}$.

Step 5.2. Selection of genetic operators.

The work of the GA is managed by three genetic operators: selection, crossover and mutation [17]. There are various modifications of these operators, so the choice of a particular variant affects the speed and quality of the solution. At this stage the probability of crossover $P_{C}$ and the probability of mutation $P_{M}$ are specified.

Step 5.3. Creation of the initial population of chromosomes. The initial population of $Z$ chromosomes is created containing information on $Z$ different variants (finite set of admissible solutions to a problem) of the values of the weigh coefficients for the given structure of $\mathrm{NC}$ network at this stage.
In turn, the initial population $P_{0}$ is described by the expression (4)

$$
P_{0}=\left\{H_{1}, H_{2}, \ldots, H_{j}, \ldots, H_{Z}\right\},
$$

where $H_{j}$ is the population's $j$-th chromosome; $Z$ is a size of population.

It is advisable to randomly set the values of the chromosomes genes in this case.

Step 5.4. Evaluation of the chromosomes of the current population. The chromosomes evaluation is carried out by the following steps.

Step 5.4.1. Decoding of chromosomes. At this stage decoding of each chromosome of the population into a set of weight coefficients of the NC network is implemented.

Step 5.4.2. Building of neural networks of the $N C$. Building of NC networks that meet the estimated chromosomes is implemented at this stage.

Step 5.4.3. Calculation of the total goal (fitness) function of the control system with NC. At this stage simulation of the control system of the MIP with developed NC is carried out and the total fitness function $J_{\Sigma}$ value is calculated for each $j$-th chromosome of the population $(j=1, . ., Z)$. Wherein, the simulation of the ACS is carried out in all possible operating modes of the MIP (at different input signals and disturbances) and the total fitness function $J_{\Sigma}$ is calculated on the basis of equation (2).

Step 5.5. Checking of the search expiration criteria. The search can expire when the following conditions occur.

Condition 1. Achievement of the optimal value of the goal function $J_{\text {Eopt }}$ of the ACS at the synthesized NC. If the fitness function value $J_{\Sigma}$ of some chromosomes reaches $J_{\Sigma \text { opt }}$ in the process of GA functioning, then the criterion is considered achieved.

Condition 2. Exceeding the maximum allowed number of iterations $n_{\max }$. The maximum number of iterations is set previously, before starting of the genetic search.

Condition 3. Degeneration of the population. Previously, before starting of the GA operating, the number of iterations $n_{p}$ and the threshold of the improvement coefficient of the fitness function values of the best chromosome $\rho_{p}$ are set. Starting from the iteration $n_{p}$, at each subsequent iteration $\left(n_{p}+1\right)$, the improvement coefficient $\rho$ is calculated and compared with the threshold value $\rho_{p}$. In turn, the coefficient of improvement $\rho$ is calculated as

$$
\rho=\frac{J_{\Sigma b e s t(n)}-J_{\Sigma b e s t(n-1)}}{J_{\Sigma b e s t(n-1)}},
$$


where $J_{\Sigma b e s t(n)}$ is the best value of the fitness function $J_{\Sigma}$ at the iteration $n ; J_{\Sigma b e s t(n-1)}$ is the best value of the fitness function $J_{\Sigma}$ at the iteration $n-1$.

If the value $\rho$ is less than $\rho_{p}$, then the stopping criterion is considered reached.

The transition to Step 5.10 is carried out in the case of occurrence of one of the given above stopping conditions.

Step 5.6. Selection of chromosomes for crossover. At this stage the population chromosomes are selected for crossover to generate new solutions on the basis of the values of the fitness function $J_{\Sigma}$. In this case, it is advisable to use the proportional selection [17], which is implemented by the following steps.

Step 5.6.1. Determination of the average value of the fitness function of the population. Determination of the average value of the fitness function $J_{\Sigma \mathrm{M}}$ of the population as the average of the arithmetic values of the fitness functions of all individuals is implemented according to equation (6)

$$
J_{\Sigma \mathrm{M}}=\frac{1}{Z} \sum_{j=1}^{Z} J_{\Sigma j}
$$

Step 5.6.2. Calculation of the selection ratio. At this stage the value of the selection ratio $P_{\mathrm{s}}(j)$ for each individual is calculated as follows

$$
P_{\mathrm{S}}(j)=\frac{J_{\Sigma j}}{J_{\Sigma \mathrm{M}}}
$$

Step 5.6.3. Forming an array of chromosomes for crossover. The array of individuals admitted to crossover is formed at this step depending on the $P_{S}(j)$ value (if $P_{S}(j)>1$, then, the individual is considered well adapted and allowed to crossover).

Step 5.7. Crossover of the chromosomes. At this stage the crossover operators for the chromosomes selected in the previous step are applied. In this case, random selection of parent couples is applied [35].

Step 5.7.1. Numbering the chromosomes. The numbering of all the representatives of the current population is implemented in an arbitrary manner at this stage.

Step 5.7.2. Choosing the first parent. At this stage the number on the interval $[0 ; 1]$ is randomly chosen for each chromosome, starting from the first one. The first parent in the pair will be the first chromosome, for which the given random number is not less than probability of crossover $P_{C}$.

Step 5.7.3. Choosing the second parent. The browsing of the population is continued, starting from the decision following after the first parent (selected in previous step), until the randomly chosen number again is not less than $P_{C}$. The chromosome for which such a condition is fulfilled will be the second parent. Step 5.7.3 is continued until the required number of pairs of parents is selected.

Step 5.7.4. Crossover of the selected parent chromosomes. At this stage the one-point crossoperator is applied for each parent pair. In turn, the crossing point in the crossover operation is chosen randomly.

Step 5.8. Mutation of chromosomes. The mutation operators are applied for chromosomes, selected at Step 5.6 according to the probability of mutation $P_{M}$ (selected at Step 5.2).

Step 5.8.1. Copying the parent chromosomes into a chromosome-heir. At this stage the parent chromosomes, admitted to mutations, are copied into the chromosomes-heirs.

Step 5.8.2. Choosing the mutant gene. The mutant gene of each chromosome, admitted to mutations, is chosen randomly.

Step 5.8.3. Choosing a new gene value. Choosing a new gene value that is not equal to the current one is implemented in a given range of permissible gene values for each mutating gene.

Step 5.9. Forming a new generation. A new generation of elite chromosomes and chromosomesheirs obtained by applying crossover and mutation are formed at this stage. Then the transition to Step 5.4 is carried out.

Step 5.10. Stopping the operation of genetic algorithm.

Step 6. Checking the criteria of the genetic search stopping. At this stage the criteria of genetic search stopping is checked and if the Condition 1 is satisfied, the transition to Step 9 is implemented. If Conditions 2 or 3 are satisfied - the transition to the next step is carried out.

Step 7. Addition of the one neuron to the last hidden layer. One neuron is added to the last hidden layer at this stage. If the maximum number of neurons in this layer is reached, then transition to the next step is carried out. Otherwise the transition to Step 5 is implemented.

Step 8. Addition of a new hidden layer. A new hidden layer is added, consisting of one neuron before the outputs layer. Provided the maximum number of hidden layers is reached, the transition to the Step 4 is carried out. Also, the return to Steps 3, 2 or 1 can be done in order to change types or parameters of fitness function and reference models, as well as inputs of the control system NC. Provided the maximum number of hidden layers is not reached, the transition to the Step 5 is implemented and genetic search starts again. 
Step 9. Implementation and the use of the developed ACS based of NC to control of complex multicoordinate interrelated plant.

To study the effectiveness of the given above neuroevolution based approach it is advisable to conduct the design process of the automatic control system for the spatial motion of multipurpose caterpillar mobile robot able to move on inclined and vertical ferromagnetic surfaces.

\section{DESIGN OF THE SPATIAL MOTION ACS FOR THE MULTIPURPOSE CATERPILLAR MOBILE ROBOT}

The multipurpose caterpillar mobile robots able to move on inclined and vertical ferromagnetic surfaces are very effective instruments for moving different working tools along given trajectories and automation of such technological operations as cleaning, rust removal, painting, welding, etc. in ship repair, shipbuilding, agriculture, gas and oil refining and in other branches of industry, where it is necessary [48-50]. The given MRs allow us to replace the monotonous human labor as well as improve the performance of technological processes and decrease the risks to workers' health and life in the life-threatening conditions [51].

However, to implement the given above operations the multipurpose caterpillar MRs have to be able to move along pre-set trajectories and operate under uncertainty of the working surface caused by their technological features, presence of obstacles, structural damage, etc. [49, 50]. Thus, automatic control of the spatial motion under the action of various disturbances is one of the most important and complicated automation tasks of MRs of such type [51]. This task involves the simultaneous control of two interrelated MR's variables: linear speed and steering angle. The given interrelation of variables as well as a number of features of the robot as a non-linear multicoordinate interrelated plant don't allow achieving high control efficiency at using separate conventional controllers in the MR's two-channel ACS [51]. Thus, it is expedient to use the proposed by the authors neuroevolution based approach to design the automatic control system for the spatial motion of the multipurpose mobile caterpillar robot.

The development of the spatial motion ACS is conducted for the caterpillar robot with the following main parameters: MR's loaded weight of $150 \mathrm{~kg}$, length of $1 \mathrm{~m}$, width of $0.8 \mathrm{~m}$, driving wheel radius of $0.15 \mathrm{~m}$, linear speed of movement of 0.3 $\mathrm{m} / \mathrm{s}$, two drive motors $2 \mathrm{~PB} 132 \mathrm{MH}$ and gear ratio of 105. The mathematical model of the MR consists of the following equations

$$
\begin{aligned}
& F=\frac{F_{\mathrm{TO}}+G(f \cos \gamma+\sin \gamma-\xi \cos \gamma)+m_{\mathrm{MR}} c \lambda}{\xi-f} ; \\
& \omega_{\mathrm{MR}}=V_{\mathrm{MR}} / R_{\mathrm{T}}=\left(V_{\mathrm{C} 2}-V_{\mathrm{C} 1}\right) / B ; \\
& V_{\mathrm{MR}}=\left(V_{\mathrm{C} 2}+V_{\mathrm{C} 1}\right) / 2 \text {; } \\
& V_{\mathrm{C} 1}=\omega_{\mathrm{MR}}\left(R_{\mathrm{T}}-0.5 B\right)=\omega_{\mathrm{W} 1} R_{\mathrm{W}} ; \\
& V_{\mathrm{C} 2}=\omega_{\mathrm{MR}}\left(R_{\mathrm{T}}+0.5 B\right)=\omega_{\mathrm{W} 2} R_{\mathrm{W}} ; \\
& R_{\mathrm{T}}=\frac{0.5 B\left(V_{\mathrm{C} 2}+V_{\mathrm{C} 1}\right)}{\left(V_{\mathrm{C} 2}-V_{\mathrm{C} 1}\right)} \text {; } \\
& M_{\mathrm{EM} a}=\frac{D_{\mathrm{M}}}{\eta_{\mathrm{M}}} \frac{d \omega_{\mathrm{M}}}{d t}+\frac{1}{k_{\mathrm{R}} \eta_{\mathrm{MR}}}\left[D_{\Sigma \mathrm{W}} \frac{d \omega_{\mathrm{W}}}{d t}+\right. \\
& +R_{\mathrm{W}}\left(f \frac{G \cos \gamma+F}{2}+G \sin \gamma\left(\frac{\cos \varphi_{\mathrm{MR}}}{2}+\right.\right. \\
& \left.+b \frac{x_{O}}{B} \sin \varphi_{\mathrm{MR}}+b f \frac{h_{\mathrm{C}}}{B} \sin \varphi_{\mathrm{MR}}\right)- \\
& -b \frac{\mu_{\mathrm{T}} L(G \cos \gamma+F)}{4 B}\left(1+\frac{4 x_{O}^{2}}{L^{2}}\right)+ \\
& +F_{\text {TO }}\left(\frac{1}{2} \cos \beta-\frac{\left(x_{1}-x_{O}\right)}{B} \sin \beta\right)+ \\
& +\left(\frac{G \cos \gamma+F}{2 g}+b G \sin \gamma \sin \varphi_{\mathrm{MR}} \frac{h_{\mathrm{C}}}{\mathrm{g} B}\right) \frac{d V_{\mathrm{C} a}}{d t}+ \\
& \left.\left.+\left(m_{\mathrm{MR}}+\frac{F}{g}\right) \frac{L^{2}+B^{2}}{12 B} \frac{d \omega_{\mathrm{MR}}}{d t}\right)\right],
\end{aligned}
$$

where $F_{\mathrm{TO}}$ and $F$ are the values of the specific forces of technological operation and the clamping magnets; $G$ is the total weight of the robot and process equipment; $\gamma$ is the surface inclination angle; $f$ is the coefficient of rolling friction; $\xi$ is the coefficient of adhesion; $m_{\mathrm{MR}}$ is the robot mass; $\lambda$ is a combined mass ratio, $\lambda=1,15+0,001 k_{\mathrm{R}}^{2} ; k_{\mathrm{R}}$ is the gear ratio; $c$ is the robot acceleration; $V_{\mathrm{C} 1}, V_{\mathrm{C} 2}$ are the current linear velocity of lagging and running caterpillars; $R_{\mathrm{T}}$ is the robot turning radius; $V_{\mathrm{MR}}, \varphi_{\mathrm{MR}}$ are the current linear speed and course of robot, $\omega_{\mathrm{MR}}$ is the rotation speed of the MR, $\omega_{\mathrm{MR}}=d \varphi_{\mathrm{MR}} / d t ; B$ is the distance between the centers of caterpillars; $\omega_{\mathrm{W} 1}$, $\omega_{\mathrm{W} 2}-$ angular velocity of lagging and running wheels; $R_{\mathrm{W}}$ is the radius of the driving wheel; $a$ and $b$ are the coefficients, that take into account direction of rotation of the robot, $a=1, b=1$ - for a lagging caterpillar; $a=2, b=-1$ - for a running caterpillar; $M_{\mathrm{EM} a}$ are the electromagnetic torques of drive motors; $\eta_{\mathrm{M}}, \eta_{\mathrm{MR}}$ are the efficiency of the motor and MR; $D_{\mathrm{M}}$ is the moment of inertia of the motor anchor; $D_{\Sigma \mathrm{W}}$ is the total moment of inertia of two 
wheels and caterpillar; $L, h_{\mathrm{C}}$ are the length of robot and height of center of gravity; $x_{O}, x_{1}$ are the distances from the transverse axis of the robot to the turning centers of the caterpillars and to the point of fixing the technological equipment; $\beta$ is the angle of deviation of the force $F_{\mathrm{TO}}$ from the longitudinal axis of the robot; $\mu_{\mathrm{T}}$ is the cornering resistance, which depends on the turning radius.

At the Step 1 of the proposed approach the functional structure of the ACS for the spatial motion of multipurpose caterpillar mobile robot is built on the basis of the single complex neural controller, which is shown in Fig. 3.

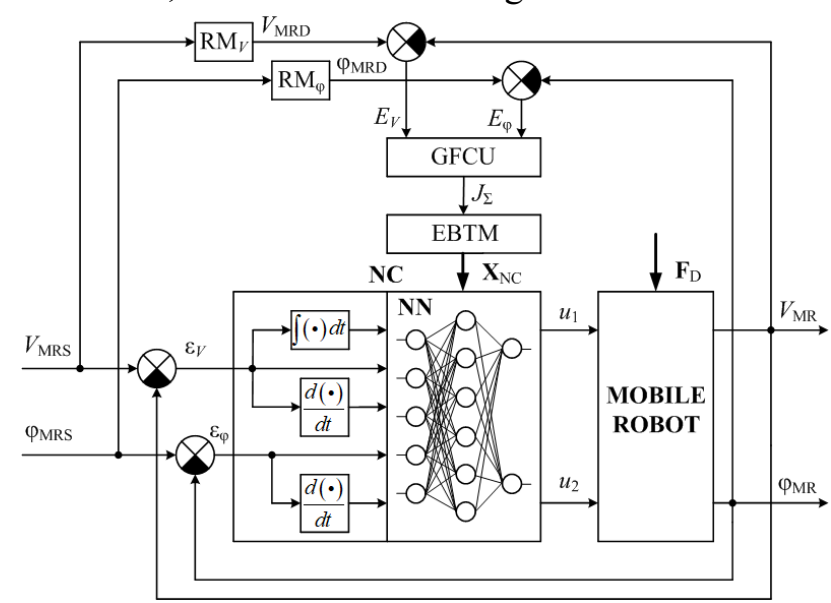

Figure 3 - Functional structure of the ACS of the MR spatial motion

In Fig. 3 the following notations are accepted: $V_{\text {MRS }}$ and $\varphi_{\text {MRS }}$ are the set values of the MR speed and course angle; $V_{\mathrm{MRD}}$ and $\varphi_{\mathrm{MRD}}$ are the desired values of the MR speed and angle, obtained at the outputs of the corresponding reference models $\mathrm{RM}_{V}$ and $\mathrm{RM}_{\varphi} ; \varepsilon_{V}$ and $\varepsilon_{\varphi}$ are the control errors of the MR variables; $E_{V}$ and $E_{\varphi}$ are the discrepancies between the RMs output signals and the real outputs of the mobile robot. The given ACS has the complex NC with 5 inputs: $\varepsilon_{V}, \dot{\varepsilon}_{V}, \int \varepsilon_{V} d t, \varepsilon_{\varphi}, \dot{\varepsilon}_{\varphi}$ and 2 outputs: $u_{1}$ and $u_{2}$, that are, in turn, the control signals of the robots corresponding caterpillars.

At Step 2 two reference models for the MR variables $V_{\mathrm{MR}}$ and $\varphi_{\mathrm{MR}}$ are selected, that are defined by the following transfer functions

$$
\begin{aligned}
& W_{\mathrm{RM} V}(\mathrm{~s})=\frac{V_{\mathrm{MRD}}(\mathrm{s})}{V_{\mathrm{MRS}}(\mathrm{s})}=\frac{1}{\left(T_{\mathrm{RM} V} \mathrm{~s}+1\right)^{2}} ; \\
& W_{\mathrm{RM} \varphi}(\mathrm{s})=\frac{\varphi_{\mathrm{MRD}}(\mathrm{s})}{\varphi_{\mathrm{MRS}}(\mathrm{s})}=\frac{1}{\left(T_{\mathrm{RM} \varphi} \mathrm{s}+1\right)^{2}},
\end{aligned}
$$

where $T_{\mathrm{RM} V}$ and $T_{\mathrm{RM} \varphi}$ are the time constants of the $\mathrm{RM}$ transfer functions $\left(T_{\mathrm{RM} V}=0.35 ; T_{\mathrm{RM} \varphi}=0.21\right)$.
At Step 3 the total goal function $J_{\Sigma}$ of the MR control system is specified in the form

$$
J_{\Sigma}=J_{V}+J_{\varphi}=\frac{k_{V}}{t_{\max }} \int_{0}^{t_{\max }} E_{V} d t+\frac{k_{\varphi}}{t_{\max }} \int_{0}^{t_{\max }} E_{\varphi} d t
$$

where $k_{V}$ and $k_{\varphi}$ are the coefficients, that normalize the values of the goal functions $J_{V}$ and $J_{\varphi}$ of the circuits of speed and angle control respectively $\left(k_{V}=\right.$ $\left.22 ; k_{\varphi}=1\right)$.

In turn, the optimal value of the total goal function $J_{\Sigma \text { opt }}$ is selected to be equal to 160 .

At Step 4 the initial structure and constraints of the multilayer neural network of the mobile robot $\mathrm{NC}$ are selected. In turn, the initial structure is chosen as follows: five input neurons, two neurons in the single hidden layer and two output neurons (52-2). In this case, the GA chromosome of minimal size consists of 14 genes. As for the constraints, the maximum possible number of hidden layers is 5 and the maximum possible number of neurons in the one hidden layer is 4 . Thus, the maximum chromosome size is 92 genes allowing us to implement the synthesis process of the network synaptic weights with acceptable computational and time costs. Also, linear activation functions are selected for two output neurons and hyperbolic tangent sigmoid activation functions are selected for other neurons at this step.

Further training of the complex $\mathrm{NC}$ of the MR spatial motion ACS in order to find the optimal structure and parameters of the multilayer neural network is implemented by the evolutionary based training mechanism at the next remaining steps of the approach. In turn, the sequential growth of layers and neurons is conducted according to Steps 7 and 8 as well as genetic search of the optimal connections weights is carried out according to Step 5. The number of chromosomes $Z$ in the population is selected equal to 150 . The maximum allowed number of iterations $n_{\max }=100$.

As a result of the conducted training the optimal structure of the NC network is found as follows: five input neurons, the first and the second hidden layers consist of four and three neurons, as well as two output neurons (5-4-3-2). For the given structure each GA chromosome consists of 38 genes and the total goal (fitness) function value $J_{\Sigma}=159.33$ is achieved at the 87-th iteration. The characters of change of the total goal function (17) value in the process of neural controller training for the obtained structure are presented in Fig. 4. 


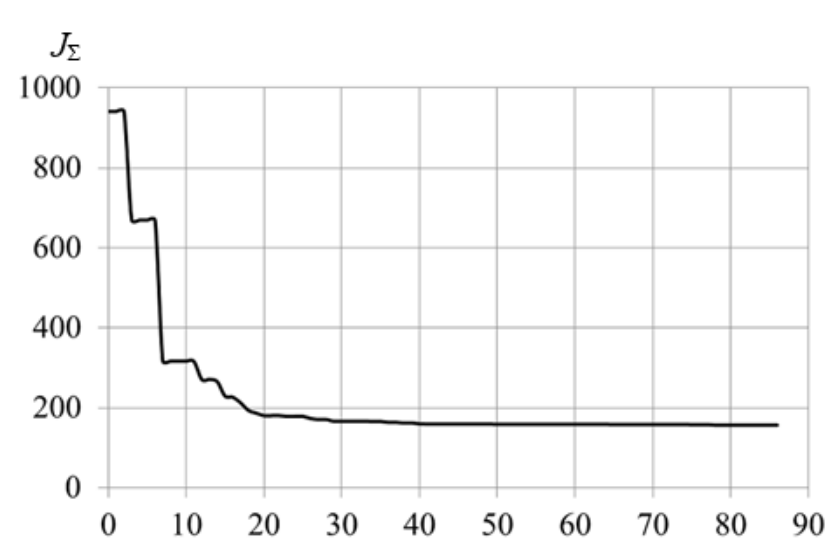

Figure 4 - The nature of the best goal function value change in the process of $\mathrm{NC}$ training

The simulation of the designed spatial motion ACS with NC of the caterpillar robot on the basis of the neuroevolutionary approach transients is conducted to study its efficiency. The comparison of the obtained simulation results is performed for two control coordinates $V_{\mathrm{MR}}$ and $\varphi_{\mathrm{MR}}$ at the output of MR's model (Fig. 3) with desired $V_{\text {MRD }}$ and $\varphi_{\text {MRD }}$ values formed at the output of their RMs as well as with traditional separate speed PID-controller and angle PD-controller in interrelated two-channels structure of MR's spatial motion ACS. In turn, the conventional speed PID-controller and angle PDcontroller are tuned via parametric optimization of basic control quality indicators with the help of gradient method. The obtained coefficients values are as follows: $k_{P V}=6.5 ; k_{I V}=422.4 ; k_{D V}=0.23$ for the speed PID-controller and $k_{P \varphi}=48.5 ; k_{D \varphi}=$ 5.2 - for the angle PD-controller. In this case, the disturbance is simulated as the constantly acting on the mobile robot maximum permissible load force from the technological operation $F_{\mathrm{TO}}=1000 \mathrm{~N}$.

The graphs of the ACS transients for step up changes of set points $V_{\text {MRS }}=0.3 \mathrm{~m} / \mathrm{s}$ and $\varphi_{\text {MRS }}=\pi / 3$ $\mathrm{rad}$ at the surface inclination angle $\gamma=60^{\circ}$ are presented in Fig. 5 and 6 respectively.

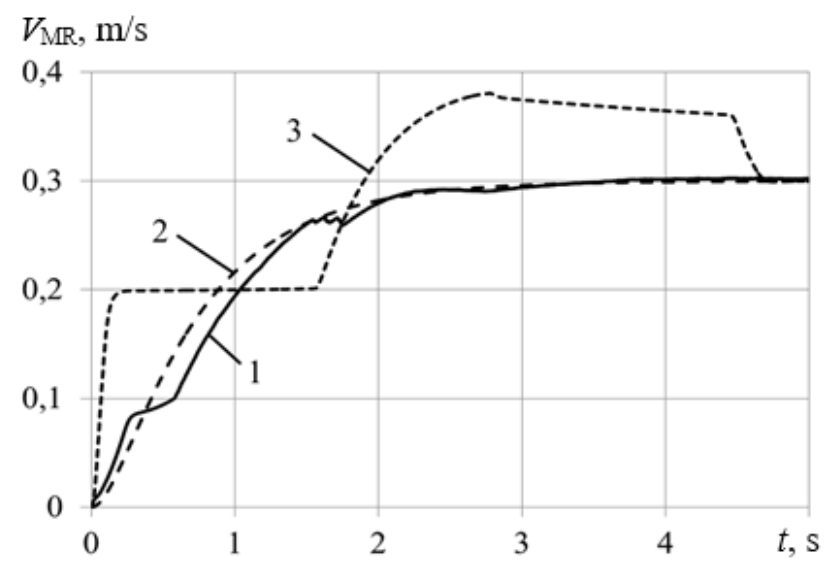

Figure 5 - ACS step up transients of MR's speed: 1 with NC of spatial movement; 2 - speed RM; 3 - with conventional PID-controller
$\varphi_{\mathrm{MR}}, \mathrm{rad}$

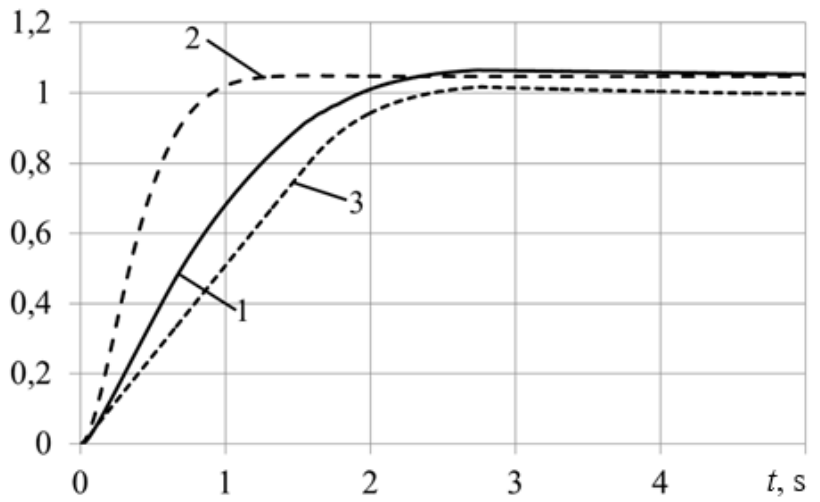

Figure 6 - ACS step up transients of MR's angle: 1 with NC of spatial movement; 2 - angle RM; 3 - with conventional PD-controller

The quality indicators comparative analysis of the spatial motion ACS of the MR with the developed NC and optimally tuned conventional controllers is presented in Table 1 , where $t_{\mathrm{t}}$ is the transient time; $\Delta$ is the static error.

Table 1. Comparative analysis of quality indicators of the ACS of the MR spatial motion (step up transients).

\begin{tabular}{|c|c|c|c|c|}
\hline \multirow{2}{*}{ Model type } & \multicolumn{5}{|c|}{ ACS quality indicators } \\
\cline { 2 - 5 } & $\boldsymbol{t}_{\mathbf{t}}, \mathbf{s}$ & $\boldsymbol{\Delta , \%}$ & $\boldsymbol{J}_{\boldsymbol{V}}$ & $\boldsymbol{J}_{\boldsymbol{\varphi}}$ \\
\hline $\mathrm{RM}_{V}$ & 2,11 & 0 & 0 & - \\
\hline $\begin{array}{c}\text { Speed with PID- } \\
\text { controller }\end{array}$ & 4,6 & 1 & 22,7 & - \\
\hline Speed with NC & 2,09 & 0,7 & 0,8 & - \\
\hline $\mathrm{RM}_{\varphi}$ & 0,89 & 0 & - & 0 \\
\hline $\begin{array}{c}\text { Angle with PD- } \\
\text { controller }\end{array}$ & 2,36 & 4,8 & - & 292,4 \\
\hline Angle with NC & 1,91 & 0,6 & - & 138,1 \\
\hline
\end{tabular}

As it can be seen in Table 1, Fig. 5 and Fig. 6 the developed ACS of the MR spatial motion on the basis of the proposed neuroevolutionary approach has considerably higher quality indicators for speed and turning angle control compared with the use of conventional ACS with separate controllers of the given variables. In particular, the use of the multicoordinate interrelated ACS with the single complex NC allows us to reduce the transients time of the speed and angle control by $55 \%$ and $19 \%$ respectively, to eliminate unwanted overshoot of $27 \%$ at speed control and significantly reduce $(8$ times) the static error at angle control. Wherein, the static error of the MR's speed control is less than 1\% that isn't critical while performing various technological operations.

The graphs of the ACS transients for step down changes of set points $V_{\mathrm{MRS}}=0.1 \mathrm{~m} / \mathrm{s}$ and $\varphi_{\mathrm{MRS}}=$ $\pi / 12 \mathrm{rad}$ (from $0.3 \mathrm{~m} / \mathrm{s}$ and $\varphi_{\mathrm{MRS}}=\pi / 3 \mathrm{rad}$ respectively) at the same simulation conditions are presented in Fig. 7 and 8. 


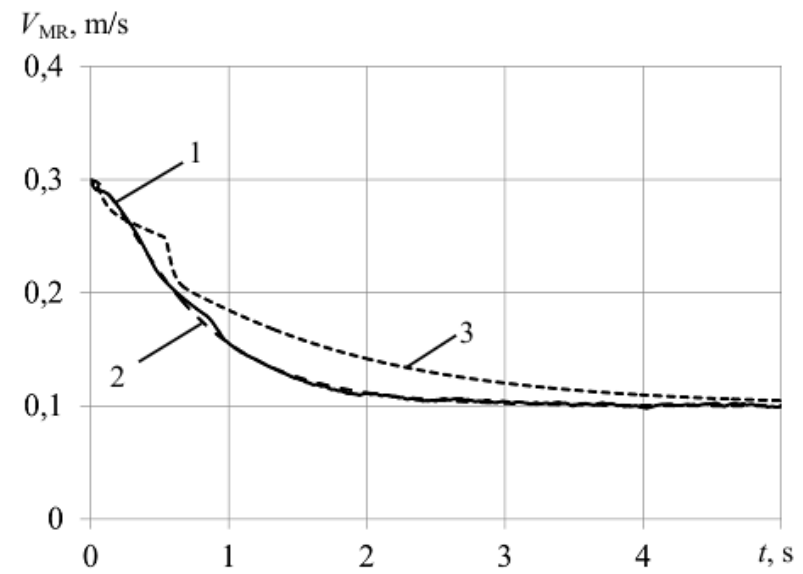

Figure 7 - ACS step down transients of MR's speed: 1 - with NC of spatial movement; 2 - speed RM; 3 - with conventional PID-controller

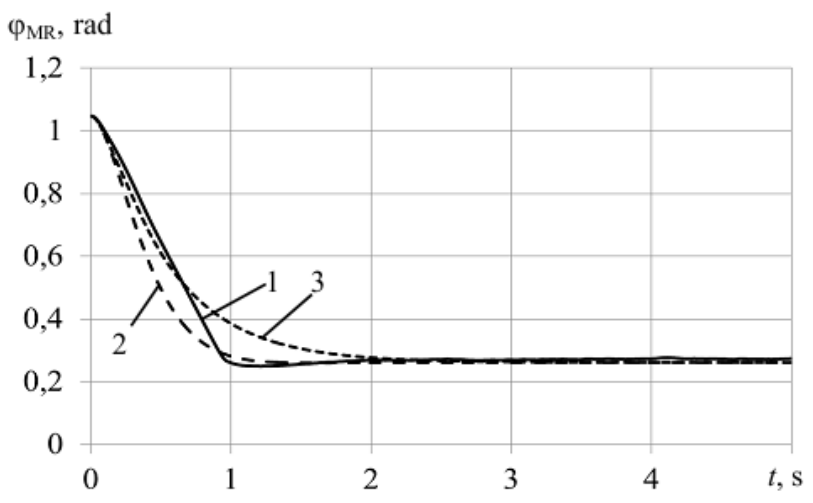

Figure 8 - ACS step down transients of MR's angle: 1 - with NC of spatial movement; 2 - angle RM; 3 - with conventional PD-controller

As well, the quality indicators comparative analysis of these transients is presented in Table 2.

Table 2. Comparative analysis of quality indicators of the ACS of the MR spatial motion (step down transients).

\begin{tabular}{|c|c|c|c|c|}
\hline \multirow{2}{*}{ Model type } & \multicolumn{4}{|c|}{ ACS quality indicators } \\
\cline { 2 - 5 } & $\boldsymbol{t}_{\mathbf{t}, \mathbf{S}}$ & $\boldsymbol{\Delta , \%}$ & $\boldsymbol{J}_{\boldsymbol{V}}$ & $\boldsymbol{J}_{\boldsymbol{\varphi}}$ \\
\hline $\mathrm{RM}_{V}$ & 2,55 & 0 & 0 & - \\
\hline $\begin{array}{c}\text { Speed with PID- } \\
\text { controller }\end{array}$ & 4,9 & 0 & 2,36 & - \\
\hline Speed with NC & 2,4 & 1 & 0,13 & - \\
\hline $\mathrm{RM}_{\varphi}$ & 1,06 & 0 & - & 0 \\
\hline $\begin{array}{c}\text { Angle with PD- } \\
\text { controller }\end{array}$ & 2,07 & 0 & - & 12,7 \\
\hline Angle with NC & 0,95 & 2,9 & - & 9,96 \\
\hline
\end{tabular}

As it can be seen in Table 2, Fig. 7 and Fig. 8 the developed ACS of the MR spatial motion on the basis of the proposed neuroevolutionary approach also has higher quality indicators for speed and turning angle control compared with the use of conventional ACS with separate controllers of the given variables. In particular, the use of the multicoordinate interrelated ACS with the single complex NC allows us to reduce the transients time of the speed and angle control by $51 \%$ and $54 \%$ respectively for step down transients, wherein the static errors at speed (1\%) and angle (2.9\%) control are not critical while performing various operations. Besides, $J_{V}$ and $J_{\varphi}$ are less for developed system in both cases of transients (step up and step down).

Thus, the synthesized ACS of the MR spatial motion with NC shows higher quality of control, better takes into account the mutual influence of control channels of the angle and speed of mobile robot than separate conventional regulators, and, accordingly, allows increasing performance and quality of the specified technological operations.

Therefore, the above studies confirm the high efficiency of the proposed neuroevolution based approach to design of control systems for complex multicoordinate interrelated plants. The application of the developed approach at designing ACSs for complex MIPs gives the opportunity to take into account the mutual influence of their control variables and, as a result, significantly improve the quality indicators of control and total effectiveness providing optimal operating modes.

\section{CONCLUSIONS}

Neuroevolution based approach to design of automatic control systems for complex multicoordinate interrelated plants, developed by the authors, is presented in this paper.

The proposed approach allows us to build the structure of the control system for the multicoordinate interrelated plant on the basis of the single complex neural controller with multiple inputs and outputs as well as to conduct the proper training of its multilayer neural network by means of the evolutionary based training mechanism, taking into account the mutual influence of all variables of the MIP in an optimal way. In particular, the given approach applies sequential growth of layers and neurons to determine the optimal structure of the NC as well as genetic algorithm to find the optimal values of its connections weights. All this gives the opportunity to implement interconnected control of the MIP variables with rather high quality indicators as well as improve its total effectiveness in the optimal structure of the developed system NC.

For studying the effectiveness of the presented approach the design process of the automatic control system for the spatial motion of multipurpose caterpillar mobile robot able to move on inclined and vertical ferromagnetic surfaces is carried out in this work. The developed ACS has a single neural controller with optimal structure and allows us to 
achieve high quality indicators of spatial motion control, taking into account the mutual influence of control channels of the MR's speed and angle, and, consequently, increase performance and quality of the specified technological operations that generally confirms the high efficiency of the proposed neuroevolutionary approach.

Further research should be related towards the practical application of the proposed neuroevolutionary based approach at the development of the software and hardware of the designed ACS with $\mathrm{NC}$ for the experimental model of the multipurpose caterpillar mobile robot able to move on inclined and vertical ferromagnetic surfaces.

\section{REFERENCES}

[1] B.R. Mehta, Y.J. Reddy, "Chapter 7 - SCADA systems," Industrial Process Automation Systems, pp. 237-300, 2015.

[2] Y.P. Kondratenko, O.V. Kozlov, O.V. Korobko, A.M. Topalov, "Internet of Things approach for automation of the complex industrial systems," Proceedings of the 13th International Conference ICTERI'2017, CEUR-WS, Ermolayev, V. et al. (Eds), Vol1844, Kyiv, Ukraine, 2017, pp. 3-18.

[3] H. Merz, T. Hansemann, C. Hübner, "Building automation: communication systems with EIB/KNX, LON and BACnet," Signals and Communication Technology, Berlin, Heidelberg: Springer-Verlag, 2009, 308 p.

[4] Y.P. Kondratenko, O.V. Kozlov, O.S. Gerasin, A.M. Topalov, O.V. Korobko, "Automation of control processes in specialized pyrolysis complexes based on Web SCADA systems," Proceedings of the 9th IEEE International Conference on Intelligent Data Acquisition and Advanced Computing Systems: Technology and Applications (IDAACS), vol. 1, Bucharest, Romania, pp. 107-112, 2017.

[5] J. Kacprzyk, Multistage Fuzzy Control: A Prescriptive Approach, John Wiley \& Sons, Inc., New York, NY, USA, 1997.

[6] B. Ross, J. Bares, C. Fromme, "A semiautonomous robot for stripping paint from large vessels," Int. J. of Robotics Research, pp. 617626, 2008.

[7] Y.P. Kondratenko, O.V. Kozlov, "Mathematic modeling of reactor's temperature mode of multiloop pyrolysis plant," Modeling and Simulation in Engineering, Economics and Management, Lecture Notes in Business Information Processing, vol. 115, pp. 178-187, 2012.
[8] B. Siciliano, O. Khatib (Eds), Springer Handbook of Robotics. Springer, 2016.

[9] M. W. Spong, S. Hutchinson, M. Vidyasagar, Robot Modeling and Control, New York: Publisher Wiley, 2006.

[10] N. K. Bose, "Applied multidimensional systems theory," Springer International Publishing AG, Springer, Cham, 2017, 192 p.

[11] E. Rogers, K. Galkowski, W. Paszke, K. L. Moore, P. H. Bauer, L. Hladowski, P. Dabkowski, "Multidimensional control systems: case studies in design and evaluation," Multidimensional Systems and Signal Processing, vol. 26, issue 4, pp. 895-939, 2015.

[12] D. Souto, A. Faiña, F. Lypez-Peca, R. J. Duro, "Lappa: a new type of robot for underwater non-magnetic and complex hull cleaning," IEEE Int. Conf. on Robotics and Automation (ICRA), Karlsruhe, 2013, pp. 3394-3399.

[13] L. Christensen, N. Fischer, S. Kroffke, J. Lemburg, R. Ahlers, "Cost-effective autonomous robots for ballast water tank inspection," J. of Ship Production and Design, August, vol. 27, no. 3, pp. 127-136, 2011.

[14] M.T. Hayajneh, S.M. Radaideh, I.A. Smadi, "Fuzzy logic controller for overhead cranes," Engineering Computations, vol. 23, issue 1, pp. 84-98, 2006.

[15] L.A. Zadeh, A.M. Abbasov, R.R. Yager, S.N. Shahbazova, M.Z. Reformat, Eds., "Recent developments and new directions in soft computing," STUDFUZ 317, Cham: Springer, 2014, $466 \mathrm{p}$.

[16] M. Jamshidi, V. Kreinovich, J. Kacprzyk, Eds., "Advance trends in soft computing," STUDFUZ 312, Cham: Springer-Verlag, 2013, $468 \mathrm{p}$.

[17] S.O. Subbotin, A.O. Oliinyk, O.O. Oliinyk, Non-iterative, Evolutionary and Multi-agent Methods for Synthesizing Fuzzy and Neural Network Models, Zaporizhzhia: ZNTU, 2009. $375 \mathrm{p}$.

[18] A. Piegat, "Fuzzy modeling and control," Vol. 69, Physica, 2013, $704 \mathrm{p}$.

[19] Z. Xiao, J. Guo, H. Zeng, P. Zhou, S. Wang, "Application of fuzzy neural network controller in hydropower generator unit," J. Kybernetes, vol. 38, no. 10, pp. 1709-1717, 2009.

[20] Y.P. Kondratenko, J. Rudolph, O.V. Kozlov, Y.M. Zaporozhets, O.S. Gerasin, "Neuro-fuzzy observers of clamping force for magnetically operated movers of mobile robots," Technical Electrodynamics, no. 5, pp. 53-61, 2017. (in Ukrainian) 
[21] R. Hampel, M. Wagenknecht, N. Chaker, "Fuzzy Control: Theory and Practice," New York: Physika-Verlag, Heidelberg, 2000.

[22] Y. Zhang, Ch. Yingliu, X. Song, Zh. Yan, "Application of RBF neural network PID controller in the rectification column temperature control system," Proceedings of the Sixth Int. Symp. on Computational Intelligence and Design, vol. 2, 2013, pp. 7275.

[23] A.I. Glushchenko, V.A. Petrov, "On neural tuner application to adjust speed P-controller of rolling mill main DC drive," Proceedings of the International Siberian Conference on Control and Communications (SIBCON), 2017, pp. 1-5.

[24] L.U. Emaletdinova, A.N. Kabirova, "Neural fuzzy controller to control the angle of heel and the course of the unmanned aerial vehicle," Proceedings of the International Conference on Dynamics of Systems, Mechanisms and Machines (Dynamics), 2016, pp. 1-5.

[25] J.K. Molina, M.J. Dominguez, C.U. Onate, H.T. Salamea, "Development of a neural controller applied in a 5 DOF robot redundant," IEEE Latin America Transactions, vol. 12, issue 2, pp. 98-106, 2014.

[26] W. Yaoyao; L. Tianlin; D. Yian, "Learning to chase a ball efficiently and smoothly for a wheeled robot," Proceedings of the 24th International Conference on Mechatronics and Machine Vision in Practice (M2VIP), Auckland, New Zealand, Nov 21-23, 2017, p. 36-41.

[27] B.M. Zaineb, A. Aicha, B.H. Mouna, S. Lassaad, "Speed control of DC motor based on an adaptive feed forward neural IMC controller," Proceedings of the 2017 Int. Conf. on Green Energy Conversion Systems (GECS), 2017, pp. 1-7.

[28] S.K. Suman, M.K. Gautam, R. Srivastava, V.K. Giri, "Novel approach of speed control of PMSM drive using neural network controller," Proceedings of the 2016 International Conference on Electrical, Electronics, and Optimization Techniques (ICEEOT'2016), 2016, pp. 2780-2783.

[29] M. Mohammadi, A. Nikbakht, A. Bavalishoar, "Fuel oil leak detection in power plant with recurrent neural network and execute in programmable logic controller," Proceedings of the 2015 2nd Int. Conf. on Knowledge-Based Engineering and Innovation (KBEI), 2015, pp. 927-932.

[30] S. Kamalasadan, G.D. Swann, R. Yousefian, "A novel system-centric intelligent adaptive control architecture for power system stabilizer based on adaptive neural networks," IEEE
Systems Journal, vol. 8, issue 4, pp. 1074-1085, 2014.

[31] Y.I. Eremenko, A.I. Glushchenko, A.V. Fomin, "On PI-Controller neural tuner implementation in programmable logic controller to improve rejection of disturbances effecting heating plant," Proceedings of the 2017 International Conference on Industrial Engineering, Applications and Manufacturing (ICIEAM), 2017, pp. 1-5.

[32] T. Sunil, M. Jayachandra, R. Prasad, "Control of ship's roll using active fin stabilizers and neural network controller," Proceedings of the 2016 Int. Conf. on Advanced Communication Control and Computing Technologies (ICACCCT), 2016, pp. 516-519.

[33] B. Xu, Ch. Yang, Y. Pan, "Global neural dynamic surface tracking control of strictfeedback systems with application to hypersonic flight vehicle," IEEE Transactions on Neural Networks and Learning Systems, vol. 26, issue 10, pp. 2563-2575, 2015.

[34] E. Bejar and A. Moran, "Deep reinforcement learning based neuro-control for a twodimensional magnetic positioning system," 4th International Conference on Control, Automation and Robotics (ICCAR), Auckland, 2018, pp. 268-273.

[35] D. Simon, Evolutionary Optimization Algorithms: Biologically Inspired and Population-Based Approaches to Computer Intelligence, John Wiley \& Sons, 2013, 772 p.

[36] A. Smiley, D. Simon, "Evolutionary optimization of atrial fibrillation diagnostic algorithms," Int. J. of Swarm Intelligence, vol. 2, nos. 2/3/4, pp. 117-133, 2016.

[37] K.O. Stanley, J. Clune, J. Lehman, R. Miikkulainen, "Designing neural networks through neuroevolution," Nature Machine Intelligence 1, pp. 24-35, 2019.

[38] S. Rodzin, O. Rodzina, L. Rodzina, "Neuroevolution: Problems, algorithms, and experiments," Proceedings of the 2016 IEEE 10th Int. Conf. on Application of Information and Communication Technologies (AICT), 2016, pp. 1-4.

[39] J. Bower, M. Shahverdi, D. Blekhman, "Neuroevolution based optimization of hybrid transmission shift points," Proceedings of the 2018 IEEE Conference on Technologies for Sustainability (SusTech), 2018, pp. 1-5.

[40] D.V. Vargas, J. Murata, "Spectrum-diverse neuroevolution with unified neural models," IEEE Trans. on Neural Networks and Learning Systems, vol. 28, issue 8, pp. 1759-1773, 2017.

[41] M.-K. Jiau, Sh.-Ch. Huang, "Self-organizing neuroevolution for solving carpool service 
problem with dynamic capacity to alternate matches," IEEE Transactions on Neural Networks and Learning Systems, vol. 30, issue 4, pp. 1048-1060, 2019.

[42] S.K. Oh, W. Pedrycz, "The design of hybrid fuzzy controllers based on genetic algorithms and estimation techniques," J. Kybernetes, vol. 31, no. 6, pp. 909-917, 2002.

[43] M. Colby, L. Yliniemi, P. Pezzini, D. Tucker, K.M. Bryden, K. Tume, "Multiobjective neuroevolutionary control for a fuel cell turbine hybrid energy system," Proceedings of the Genetic and Evolutionary Computation Conference GECCO'16, Denver, Colorado, USA, 2016, pp. 877-884.

[44] J. Gea, F. Kirchner, "Using neuroevolution for optimal impedance control," Proceedings of the 2008 IEEE Int. Conf. on Emerging Technologies and Factory Automation, 2008, pp. 1063-1066.

[45] S.D. Prestwich, S.A. Tarim, R. Rossi, B. Hnich, "A neuroevolutionary approach to stochastic inventory control in multi-echelon systems," International Journal of Production Research, vol. 50, issue 8, pp. 2150-2160, 2012.

[46] B. Trasnea, L.A. Marina, A. Vasilcoi, C.R. Pozna, S.M. Grigorescu, "GridSim: A vehicle kinematics engine for deep neuroevolutionary control in autonomous driving," Proceedings of the 2019 Third IEEE Int. Conf. on Robotic Computing (IRC), 2019, pp. 443-444.

[47] N.K. Bose, B. Buchberger, J.P. Guiver, Multidimensional Systems Theory and Applications, Berlin: Springer, 2003, 268 p.

[48] D. Souto, A. Faiña, A. Deibe, F. Lopez-Peña, R. J. Duro, "A robot for the unsupervised gritblasting of ship hulls," Int. J. of Advanced Robotic Systems, vol. 9, no. 82, pp. 1-16, 2012.

[49] D. Longo, G. Muscato, "A small low-cost lowweight inspection robot with passive-type locomotion," Integrated Computer-Aided Engineering, vol. 11, pp. 339-348, 2004.

[50] Y. Kondratenko, Y. Zaporozhets, J. Rudolph, O. Gerasin, A. Topalov, O. Kozlov, "Modeling of clamping magnets interaction with ferromagnetic surface for wheel mobile robots," International Journal of Computing, vol. 17, issue 1, pp. 33-46, 2018.

[51] Y.P. Kondratenko, Y.M. Zaporozhets, J. Rudolph, O.S. Gerasin, A.M. Topalov, O.V. Kozlov, "Features of clamping electromagnets using in wheel mobile robots and modeling of their interaction with ferromagnetic plate," Proceedings of the 9th IEEE International Conference on Intelligent Data Acquisition and Advanced Computing Systems: Technology and
Applications (IDAACS), Bucharest, Romania, 2017, vol. 1, pp. 453-458.

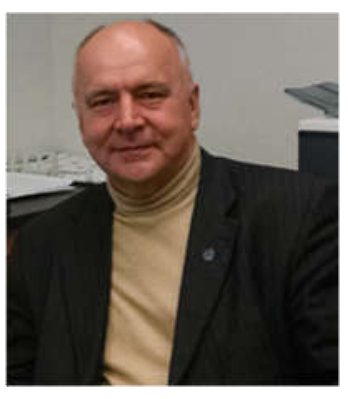

Yuriy Kondratenko, Doctor of Science, Professor, Honour Inventor of Ukraine (2008), Corr. Academician of Royal Academy of Doctors (Barce-lona, Spain), Head of the Department of Intelligent Information Systems at Petro Mohyla Black Sea National University (PMBSNU), Ukraine. He has received (a) the Ph.D. (1983) and Dr.Sc. (1994) in Elements and Devices of Computer and Control Systems from Odessa National Polytechnic University, (b) several international grants and scholarships for conducting research at Institute of Automation of Chongqing University, P.R.China (1988-1989), Ruhr-University Bochum, Germany (2000, 2010), Nazareth College and Cleveland State University, USA (2003), (c) Fulbright Scholarship for researching in USA (2015/2016) at the Dept. of Electrical Engineering and Computer Science in Cleveland State University. Research interests include robotics, automation, sensors and control systems, intelligent decision support systems, fuzzy logic.

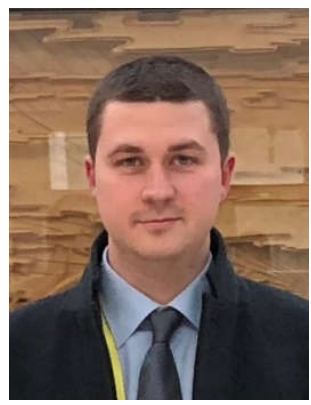

Oleksiy Kozlov, Candidate of Technical Sciences (Ph.D.), Associate Professor of the Department of Intelligent Information Systems at Petro Mohyla Black Sea National University (PMBSNU), Ukraine. $\mathrm{He}$ is a specialist in control processes automation and electrical engineering. He has received the Ph.D. in control processes automation (2014) and master degree in electromechanics (2011). His research interests include automation, intelligent control systems, robotics, fuzzy logic, elements and devices of computing systems.

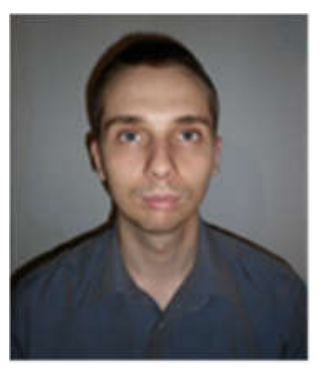

Oleksandr Gerasin, is a Junior Researcher of Admiral Makarov National University of Shipbuilding, Ukraine. $\mathrm{He}$ is specialist in control systems for electric actuators. He has gained several diplomas of bachelor and master of electromechanics. His research interests include computer control systems, modeling, fuzzy logic, intelligent robotics. 